\title{
Trajectory Data Modeling: A Mobile Hotel Use Case
}

\author{
Halgare Nanasaheb \\ Mahadev \\ Research Scholar Kalinga \\ University \\ Naya Raipur, Raipur(CG)
}

\author{
Ali Akbar Bagwan, PhD \\ Research Supervisor \\ Kalinga University \\ Chhattisgarh, India
}

\author{
D. V. Biradar \\ Head Department of \\ Information Technology \\ M. S. Bidve Engineering \\ College Latur
}

\begin{abstract}
A large amount of data resulting from trajectories of moving objects activities are collected from various localization based services and some associated automated processes. This Trajectories data can be used either for transaction and analysis purposes in various domains (commerce, environment, migration of bird, etc.). For this reason, modeling trajectory data at the conceptual level is an important step leading for successful implementations. current modeling tools fail to fulfill specific moving objects activities requirements. In this paper, it propose a new profile based on UML to enhance the conceptual modeling of trajectory data related to mobile objects by new stereotypes and icons. As illustration, it present a mobile hotal use case.
\end{abstract}

\section{General Terms}

Trajectory, trajectory model, starUML, et. al.

\section{Keywords}

Trajectory data, Conceptual modeling, positioning technologies, Location based services, Moving objects, UML extension

\section{INTRODUCTION}

In many countries rural population undergo various problems related to food necessities. In fact due to food problem they have to cross long distance to reach hotels. This, obviously, may generate life complications. Mobile hotels, which may contribute to decrease such misery, can move to populations, following declared emergencies and/or planned activities. The notion of mobile hotels was emerged then to try to decrease the geographical disparities related to food providing services It takes profits of the emergence of adapted hotel hardware, positioning technologies and pervasive systems. In fact, technologies of mobile communications and ubiquitous computing are saturating it new world. Wireless networks are becoming the nerves of it territory; through these nerves, the movement of people and vehicles may be sensed and possibly recorded, thus producing large volumes of mobility data. Modelling

the trajectory of the mobile object, such as a mobile hotel in it case, represents real challenge. Indeed, witnessing the trajectory of a moving hotel implies the recording of different information leading to get a finite set of observations destined to current transactional and future analysis activities. The mobility of a hotel, supported by adequate terrestrial transportation mean, is constrained by the road network. Mobile hotels manager defines for each mobile hotel its trajectory which is composed by a set of stops and moves. For each trajectory, the mobile hotel is equipped with food, waiter, drivers, and a local mobile hotel manager. This latter is equipped with a PDA used to manage trajectory data. He has to indicate the beginning and the end of the trajectory, each stop and move. He also sends to or receives from messages to the responsible of the mission. waiter, equipped equally with PDA, sends data related to customer and examinations to a centralized data warehouse. Such data are important since they allow detecting prospective epidemic peaks. Modelling trajectory data at the conceptual level is very important since it leads to a global vision and successful implementations. Hoover, currents modelling tools fail to fulfil specific moving objects, such as mobile hotel, activities requirements. In this paper, it propose a new profile based on UML in order to enhance the conceptual modelling of trajectory data related to mobile objects by new stereotypes and icons. As illustration, it present a mobile hotel use case. This paper is organized as follows: In section 2, it will present different research works related to moving objects (definition of moving objects and different types of those latter), trajectory data conceptual model and positioning technologies. In section 3 , it will propose a trajectory data conceptual modelling for mobile hospitals. In section 4 , it will present the implementation of it trajectory data profile based on UML. In section 5, it will summarize the work and give some perspectives to be done in the future.

\section{STATE OF THE ART}

A moving object is considered as a spatial object which changes its location over time. It is characterized by its numerous positions, which vary in space and time, describing a trajectory. The trajectory of a moving object is considered then as a path since it reflects the evolution of an object travelling in some space during a given time interval. Some works [1,2, and 3] define a trajectory as a set of stops and moves. Each element of a set has a "begin" and an "end". The "stop" is considered as an important component of the trajectory which is characterized by a non-empty time interval. This means that the moving object can perform many actions but it has to be immovable in a given "stop". A move is delimited by two stops betiten which a moving object is in movement. Authors in [4] distinguish many types of trajectories according to relationships betiten them or with their environments. For the first type of relationship, it can cite those relations: Intersect (two trajectories cross themselves), Equal (a trajectory is near another trajectory), Near (a trajectory is near another trajectory) and Far (a trajectory is far from another trajectory). For the second type of relationship, trajectories can be in relation with other spatial objects such as infrastructure elements (roads, buildings, etc.) or virtual entities such as border of a city. Among those relationships it can cite: Stay within (the trajectory is always in a sector of interest), Bypass (the trajectory pass by the sector of interest), Leave (the trajectory leaves the sector of interest), and Enter (the trajectory enters the sector of interest) and Cross (the trajectory crosses the sector of interest). To collect data resulting from moving objects activities, positioning and sensors technologies, and services associated to them and technologies with sensors seem to be a necessity. In fact, they permit to gather, to 
process and to broadcast localization information and data generated in it. Among those technologies it can mention GPS (Global Positioning System), RFID (Radio Frequency Identification tags), Bluetooth, WiFi, ZigBee, etc., which permit the localization and the follow up of persons, goods and animals on move. Positioning technologies can be classified into two categories according to their mobility degree [5]: fixed positioning technologies and mobile ones. Positioning technologies $[5,6,7]$ can have many functions such as authentication, data transfer via smart cards for example, object localization, etc. Services associated to them are responsible of the acquisition of data for a given system which is able to collect data coming from his environment. Many research works itre interested in the conceptual modelling of data and data warehouses [8,9, and 10], spatial and temporal data and data warehouses [11, 12, and 13] but few of works itre interested in the conceptual modelling of trajectory data and trajectory data warehouses [1, 14]. In [1], the requirements for the trajectory modelling take into account the characterization of trajectories and their components and the different types of constraints (semantic, topologic, etc.) in order to fix a conceptual view of the concept of trajectory. The conceptual model is seen as a direct support and an explicit representation of trajectories' components (stops, moves, begins, ends). The two solutions proposed in [1] are driven by different modelling goals. The first solution suggests a trajectory design pattern, and the second one recommends some trajectory data types. The two approaches can be combined to offer richer modelling tools when needed. The design pattern is a predefined generic schema that can be connected to any other database schema by the designer and can be adjustable. In fact, the designer can modify the design pattern by adding new elements or semantic attributes, and/or deleting other ones to adapt it to the requirements of the new applications. The second approach is based on trajectory data types. It holds the idea that semantic information is specific to an application and cannot be encapsulated into a data type, but has to be defined by the database designer. Moreover, authors in [1] define a generic data types to hold the trajectories' components such as the "begin", the "end", the "stops" and the "moves", etc., and functions of interpolation. It have to mention that authors in [1], extended those data models for trajectories from MADS model [15], since this latter supports spatial and temporal objects and relationships. Indeed, they announce icons defined in it, in their trajectory data relational conceptual model to make it more understandable by users.

\section{TRAJECTORY DATA CONCEPTUAL MODELING \\ 3.1 Mobile hospital's states conceptual modelling}

The trajectory of the mobile hotel is composed by a set of "moves" and "stops". The mobile hotel can be in the states "ready", "move in road", "stop failure", "stop in destination" and "move in destination". It is ready when everything and everyone are in the transportation platform and the driver is ready to start his mission (to move in his trajectory). It is in "move in road" when everything and everyone are in the transportation platform which is in motion in the road network. It is "stop failure" when the transportation platform is stopped because of broke down or any other problem. It is in "stop in destination" when the mobile hotel arrives to the targeted destination which is a place belonging to mobile hospital trajectory fixed by the responsible of the mission. It is in "move in destination" when the mobile hotel moves around the destination to find some emergencies in cases of critical state of customer or to find the nearest hotel encapsulating food and/or care givers specialists that don't exist in the mobile hotel. It propose the following schema to describe the different states of the mobile hotel during his trajectory.

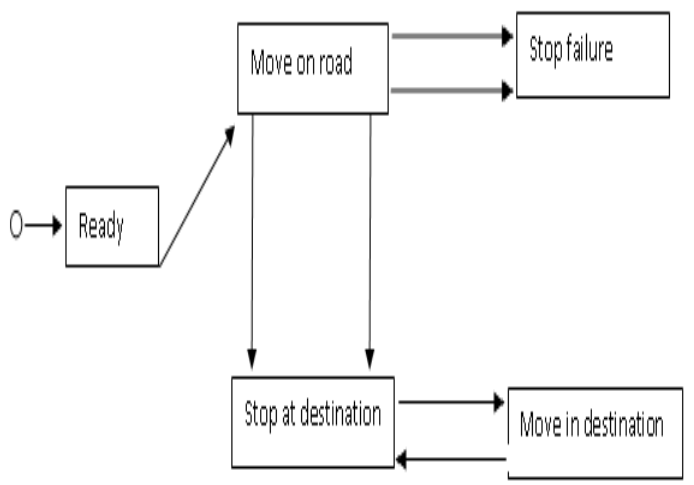

Fig.1 Different State of Mobile Hotel

\subsection{B.Hotel staff's tasks conceptual modelling}

In this section, it present some tasks which are done by the Hotel staff such as Capton. Those latter can consult the table "Customer" or add a new Customer or add new description in the table "Customer". In fact, the Capton can simply wrote the customer name that he wants to consult, the system checks the presence of this customer and display the customer requirements. Finally the system display customer information. After the dealing, the capton can add a requirements for example to the table "customer". In fact, the capton wrote the customer requirements, and then the system sends the added information to the table "customer". It propose the following sequence diagrams that illustrate the description cited above 


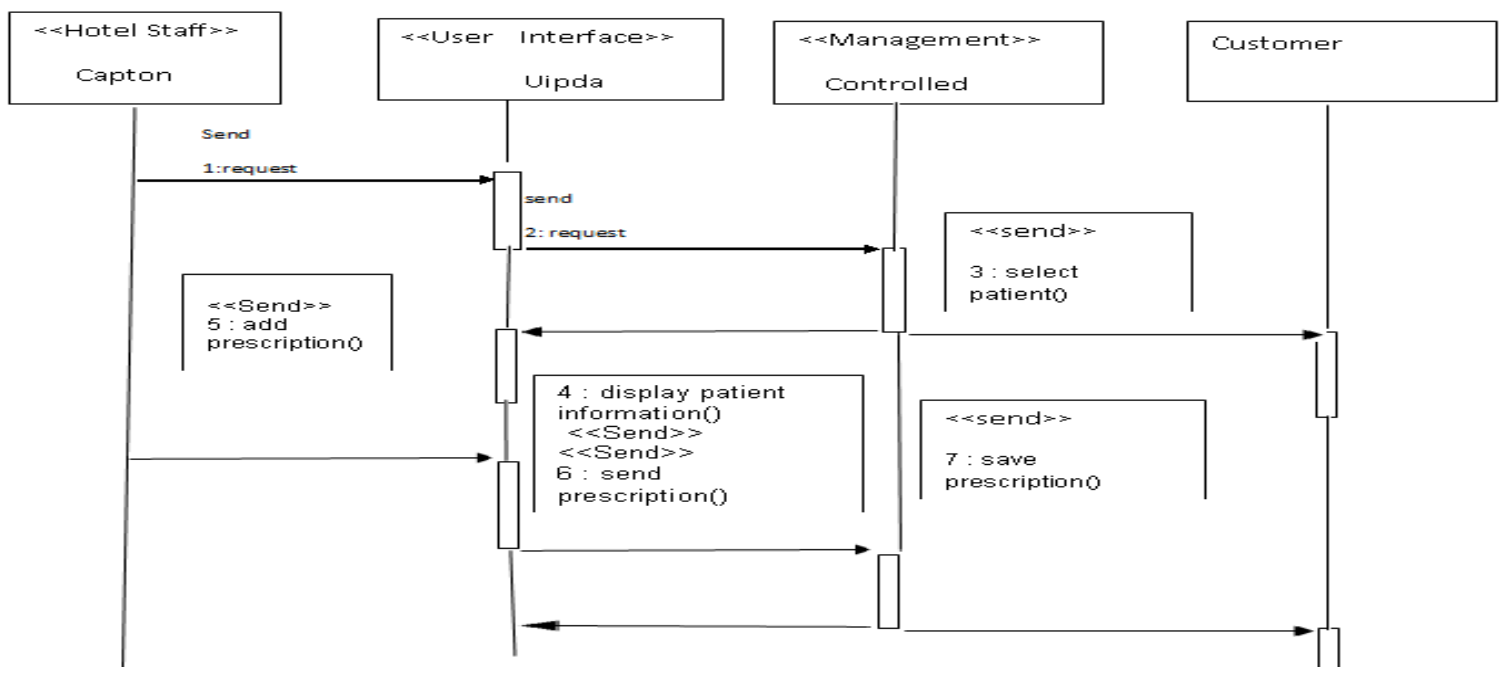

Figure 2. Consult and modify customer sequence diagram

\subsection{Mobile hotel trajectory data conceptual modelling}

- The conceptual modelling of trajectory data resulting from a mobile hotel on road is represented by a UML class diagram. In the following, it describes classes which model the trajectory data and the different relationships betiten them:

- A mobile hotel class is composed by hotel equipment and staff subclasses. Each mobile hotel has its own trajectory which is composed by a set of moves and stops.

- A Trajectory class is considered as a travel in the space and in the time. It is composed by a set of trajectory sections. It is characterized by a set of attributes such as the "begin", the "end" and the duration of a given trajectory.

- A Trajectory-section class is a component of the whole trajectory. Each trajectory section is composed by two successive "stops" and one "move".

- A Stop class is modelled by its position in the space and its time of "begin" and "end". The "begin" of a "stop" is the "end" of a previous "move". As the "stop" is the core component of the trajectory, it propose the following sequence diagram to show how to specify a given stop.

- A Move class is delimited by two successive "stops". The move is modelled by the position of the "begin" and of the "end" of a movement. Those positions are represented by absolute coordinates which are variable in time. As the move is the core component of the trajectory, it propose the following sequence diagram to show how to specify a given move in the system.

- A Customer class describes the person treated in the mobile hotel by hotel staff.

- A Capton class describes the person who is working in the mobile hotel.

- A Witer class describes the person who is working in the mobile hotel.

- A Driver class describes the person who is driving the mobile hotel.

- A GPS-data class can be connected to a given PDA and has an identifier and a set of attributes like the latitude and the longitude

- A PDA class is the mean of sending trajectory data. In fact, each manager and capton has one PDA to send trajectory data and to communicate with the responsible of his mission. The PDA has an identifier and it is connected to a GPS.

- A mobile hotel manager class describes the person who is sending trajectory data using a given PDA. The following class diagram is related to trajectory data resulting from mobile hotel. It created a new UML profile called Trajectory-UML, in which it added some pictograms and some stereotypes to identify each class (entity). It used pictograms of MADS project [15] only for some entities which are representing trajectory and trajectory components such as "move", "stop", "trsection", and "location". The same idea was done in [1] but in a relational model.

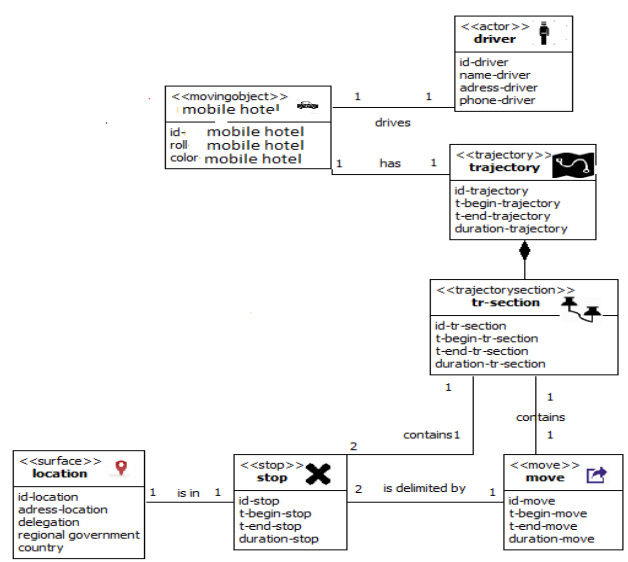

\section{REALIZATION OF TRAJECTORY UML PROFILE}

In it solution, it propose to add some extensions (stereotypes, icons) to UML diagrams (class diagram, sequence diagram). This leads to it trajectory profile which is based on UML. It itre inspired by the work of $[1,15]$ to extend the UML profile in order to bring to light the trajectory and its environment. An UML profile allows specializing UML in a precise domain. It trajectory UML profile is composed of two 
diagrams which are the trajectory data sequence diagram and the trajectory data class diagram. The first diagram has the aim to show how to consult a "customer" or to add some information to it. In the following table it describe each element of the trajectory data sequence diagram and the added stereotypes and icons.

Table 1. Stereotypes and icons of the trajectory data sequence diagram

\begin{tabular}{|l|l|l|}
\hline Elements & Stereotypes & Icon \\
\hline Capton & $<<$ Hotel staff $>$ & \\
\hline Customer & $<<$ Suffering $>>$ & \\
\hline UIpda & $<<$ Userinterface $>>$ \\
\hline Controllpda & $<<$ Management $>>$ & \\
\hline
\end{tabular}

The second diagram has the aim to show trajectory data classes and interactions batten them. In the following table, it describe it new stereotypes and icons for each class which is in interaction with the trajectory class and the moving object class.

Table II. Stereotypes and icons of the trajectory data class diagram.

\begin{tabular}{|c|c|c|}
\hline Elements & Stereotypes & Icons \\
\hline Trajectory & «trajectory» & $\begin{array}{c}\text { «trajectory- } \\
\text { section» }\end{array}$ \\
\hline Trajectory-section & «stop» & \\
\hline Stop & «move» & \\
\hline Move & & \\
\hline
\end{tabular}

\begin{tabular}{|c|c|c|}
\hline Gps & «gps data» \\
\hline Location & «surface» \\
\hline Mobile hotel & «moving object» & \\
\hline capton/waitors & «hotel staff » \\
\hline Driver/manager & «actor» & \\
\hline
\end{tabular}

To implement the trajectory data sequence diagram and the trajectory data class diagram, it used the open sitce platform called StarUml. This latter is extensible since it uses the XML. In fact, StarUML allows adding new functions which are adaptable to users' needs. It extended a new approach of UML called tdw (trajectory data warehouse) approach and a new profile. The "tdw approach" defines new types of diagrams (trajectory data sequence and class diagrams) and their order of appearance. The trajectory UML profile is used to widen the capabilities of UML to express specific elements in a certain domain. The following schema shows the "tdw approach.

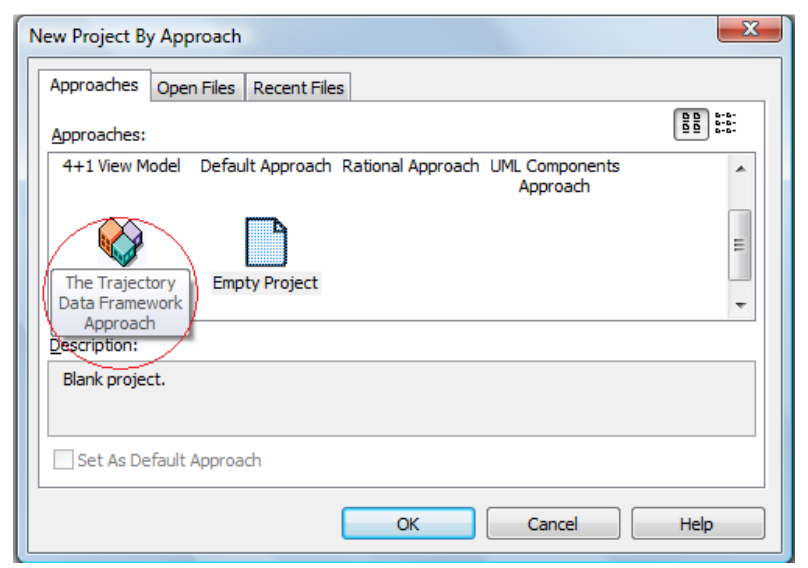

After having chosen the "tdw approach", it find in the part "Model Explorer", the added model called TDW model which consists of two sub modes called trajectory data sequence diagram and trajectory data class diagram. The following schema shows the description cited above. 


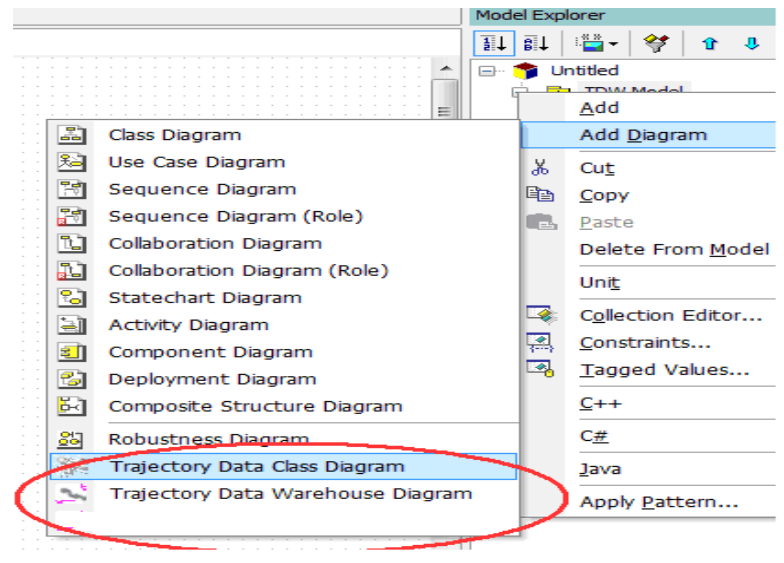

\section{CONCLUSION}

In this work, it presented concepts related to trajectory data resulting from a specific mobile object which is the mobile hotel. It proposed a new profile called trajectory profile which is based on UML. In this trajectory profile, it defined a trajectory data sequence and a trajectory data class diagrams with new stereotypes and icons to concise the conceptual design of trajectory data. Such trajectory data resulting from mobile hotel can be gathered and stored in a trajectory data warehouse then can be analyzed deeply using data mining tools to make strategic decision about some prospective epidemic peaks.6

\section{REFERENCES}

[1] S .Spaccapietra., C. Parent, M. L. Damiani, J. A. de Macedo, F. Porto, C. Vangenot. A Conceptual View on Trajectories. Research Report. Ecole Polytechnique Fédérale, Database Laboratory, Lausane, Switzerland. May, 29th, 2007.

[2] J .Fernando. Braz. "Trajectory data warehouse proposal of design and application to exploit data". IX Brazilian Symposium on GeoInformatics, Campos do Jordão, Brazil, November 25-28, 2007, INPE, p 61-72. 2007.

[3] J .Trujillo., M. Palomar et J. Gomez, I.Y. Song. "Designing data warehouses with OO conceptualmodels". IEEE Computer 34 (12) 66-75 (Special issue on Data Warehouses). 2001

[4] S .Brakatsoulas., D. Pfoser, and N. Tryfona. Modeling, storing and mining moving objects databases. In International Database Engineering and Applications Symposium (IDEAS), pp. 68-77, 2004.

[5] I.Sandu Popa,Ahmed Kharrat Karine Zeitouni. «CALM - Un système de gestion de données de capteurs à Localisation Mobile ». Conférence SAGEO. 2008.
[6] A .Bouju., F. Bertrand, V. Mallé-Noyon, S. Servigne, T. Devogele, C. Ray, H Martin et J. Gensel. « Gestion de données spatio-temporelles au sein de bases de données capteurs ». Conférence sur les Technologies de l'Information, de la Communication et de la Géolocalisation dans les Systèmes de Transports Bretagne. 2009.

[7] E. Gaudreau, Bruno Agard, Martin Trepanier et Pierre Baptiste. «Pilotage réactif des systèmes de production à l'aide de capteurs intelligents ». 6e Congrès international de génie industriel- Besançon (France). 7-10 juin 2005.

[8] S. Orlando, Renzo Orsini, Alessandra Raffaetà, Alessandro Roncato and Claudio Silvestri. "Trajectory Data Warehouses: Design and Implementation Issues". Jitnal of computing science and engineering, vol.1, no.2, pages 211-232. 2007.

[9] X .Meng. Z. Ding. "DSTTMOD: A Discrete SpatioTemporalTrajectory Based Moving Object Databases System". LNCS 2736, (Springer verlag). 2003

[10] S. Lujàn-Mora, Juan Trujillo et Il-Yeol Song. "A UML profile for multidimensional modelling in datawarehouses". Data \& Knowledge Engineering (DKE), 59(3), 725-769. 2006.

[11] G .Pestana. M. Mira da Silva. "Multidimensional Modeling Based on Spatial, Temporal and SpatioTemporal Stereotypes". ESRI International User Conference San Diego Convention Center, California. 2005.

[12] M .Miquel. Bedard Yvan. « Conception d'entrepôts de données géo spatiales à partir de sitces hétérogènes ». Exemple d'application en foresterie. Ingénierie des systèmes d'information, pages 89-111. 2002.

[13] S. Nebojsa, Han Jiaiti et Kosperski Krzysztof. "ObjectBased Selective Materialization for Efficient Implementation of Spatial Data Cubes". IEEE Transactions on Knowledge and Data Engineering, Vol. $12, \mathrm{n}^{\circ} 6, \mathrm{p}$ 938-958. 2000.

[14] W .Oueslati., J. Akaichi. Mobile information collectors' trajectory data warehouse design. International jitnal of managing information technologies. P 1-20,2010.

[15] C .Parent., S. Spaccapietra and E. Zimanyi. Conceptual Modeling for Traditional and Spatio-Temporal Applications - The MADS Approach. In Springer Verlag,2006. 